\title{
The High Energy Materials Science Beamline at PETRA III
}

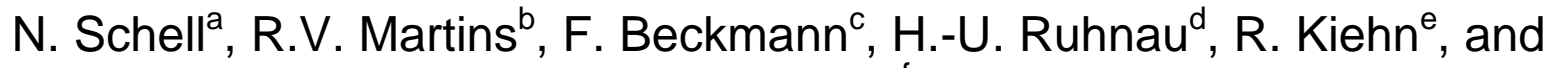 \\ A. Schreyer ${ }^{\dagger}$ \\ GKSS-Research Center Geesthacht GmbH, Max-Planck-Str. 1, 21502 Geesthacht, \\ Germany

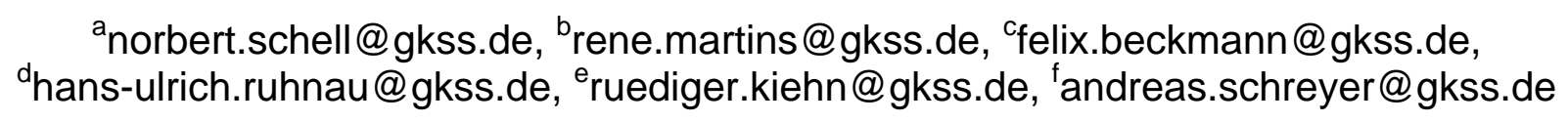

Keywords: beamline instrumentation, synchrotron radiation, high energy $\mathrm{x}$-rays

\begin{abstract}
The future High Energy Materials Science Beamline HEMS at the new German high brilliance synchrotron radiation storage ring PETRA III [1] will have a main energy of $120 \mathrm{keV}$, will be fully tunable in the range of 50 to $300 \mathrm{keV}$, and will be optimized for sub-micrometer focusing with Compound Refractive Lenses and Kirkpatrick-Baez Multilayer mirrors. Design and construction is the responsibility of the Research Center Geesthacht, GKSS, with approximately $70 \%$ of the beamtime being dedicated to Materials Research, the rest reserved for "general physics” experiments covered by DESY, Hamburg.

Fundamental research will encompass metallurgy, physics and chemistry. For first experiments in investigating grain-grain-interactions a dedicated 3D-microstructure-mapper will be designed. Applied research for manufacturing process optimization will benefit from the high flux in combination with ultra-fast detector systems allowing complex and highly dynamic in-situ studies of microstructural transformations. The beamline infrastructure will allow easy accommodation of large user provided equipment. Experiments targeting the industrial user community will be based on well established techniques with standardised evaluation, allowing "full service" measurements. Environments for strain mapping [2] on large structural components up to $1 \mathrm{t}$ will be provided as well as automated investigations of large numbers of samples, e.g. for tomography and texture determination.

The current design for the beamline (P07 in sector 5 of the future experimental hall) consists of a nearly five meter in-vacuum undulator source (U19-5) optimized for high energies, a general optics hutch, an in-house test facility and three independent experimental hutches working alternately, plus additional set-up and storage space for long-term experiments. HEMS should be operational in spring 2009 as one of the first beamlines running at PETRA III.
\end{abstract}

\section{Introduction}

PETRA III will be the new high-brilliance synchrotron radiation source on the DESY site in Hamburg-Bahrenfeld. For a total investment of 225 million $€$, to be shared by the German Federal Government (90\%) and the City of Hamburg (10\%), the existing storage ring PETRA will be converted into one of the most brilliant x-ray sources worldwide with 14 new beamlines and up to 30 experimental stations [3]. After starting the conceptual design in 2002 and the final approval of the project in May 2005, the reconstruction of the storage ring began July 2, 2007.

Several organisations will collaborate to build the various beamlines and enhance the planned infrastructure at the site. Design, construction, operation and main funding of the future High Energy Materials Science Beamline HEMS is the responsibility of the Research Center 
Geesthacht, GKSS [4]. Approximately $70 \%$ of the beamtime will be dedicated to Materials Research, the rest reserved for "general physics" experiments covered by DESY.

Fundamental research will encompass metallurgy, physics, chemistry, biology. First experiments are planned for the investigation of the relation between macroscopic and microstructural properties of polycrystalline materials, grain-grain-interactions, re-crystallisation processes, and the development of new and smart materials or processes. Applied research for manufacturing process optimization will benefit from the high flux in combination with ultra-fast detector systems allowing complex and highly dynamic in-situ studies of micro-structural transformations, e.g. during friction stir welding. Experiments targeting the industrial user community will be based on well established techniques with standardised evaluation, allowing "full service" measurements. Environments for strain mapping will be provided as well as automated investigations of large sample numbers, e.g. for texture determination and tomography.

In the following we describe the expected characteristics, the beamline layout and the current design of various planned experiments.

\section{Beamline Characteristics}

The source. The basic design parameters of the storage ring PETRA are an energy of $6 \mathrm{GeV}$, a current of $100 \mathrm{~mA}$, and an emmittance of $1 \mathrm{nmrad}$ (horizontally) and $0.01 \mathrm{nmrad}$ (vertically), respectively. The source for HEMS will be a $4.5 \mathrm{~m}$ long in-vacuum undulator (U19-5) at a high- $\beta$ position optimized for full tunability from $30 \mathrm{keV}$ onwards. The key parameters chosen are shown in Fig. 1 together with the resulting spectra (priv. comm. M. Tischer, DESY).

$$
\begin{aligned}
& \sigma_{\mathrm{x}}=140 \mu \mathrm{m} \quad \sigma_{\mathrm{x}}{ }^{\prime}=7.0 \mu \mathrm{rad} \\
& \sigma_{\mathrm{v}}=4.9 \mu \mathrm{m} \quad \sigma_{\mathrm{v}}{ }^{\prime}=2.0 \mu \mathrm{rad} \\
& \sigma_{\mathrm{r}}=1.2 \mu \mathrm{m} \quad \sigma_{\mathrm{r}}{ }^{\prime}=1.6 \mu \mathrm{rad} \\
& \rightarrow \text { fully emittance limited beam }
\end{aligned}
$$

\section{Minimum gap $=7.0 \mathrm{~mm}$}

$\lambda_{U}=19 \mathrm{~mm}$

$\mathrm{B}_{\mathrm{o}}=0.70 \mathrm{~T}$

$\mathrm{L}=4.5 \mathrm{~m}$

$\mathrm{E}_{1}=10.2 \mathrm{keV}$

$\mathrm{dP} / \mathrm{d} \Omega=0.25 \mathrm{~W} / \mu \mathrm{rad}^{2} \quad \mathrm{P}_{\text {tot }}=5.6 \mathrm{~kW}$
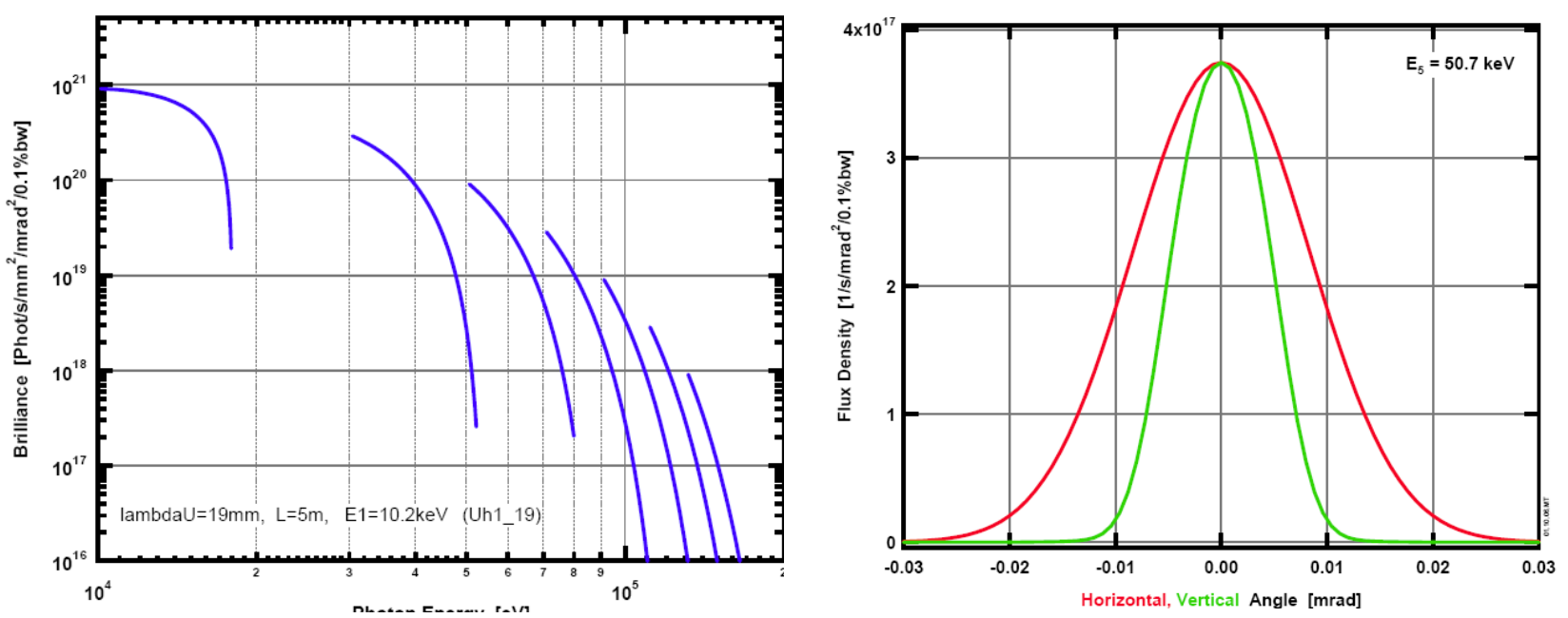

Fig. 1: Source parameters and resulting brilliance and flux density, respectively, for HEMS.

Floorplan. Due to already quite detailed demands for future experiments as discussed in a first HEMS workshop in June 2006, the current working design for the beamline (P07 in sector 5 of the $280 \mathrm{~m}$ long experimental hall) consists of a main optics hutch (OH1), an in-house test facility (EH1) and three independent experimental hutches (EH2, EH3 and EH4) working alternately, plus 
additional focussing optics hutches $(\mathrm{OH} 2, \mathrm{OH} 3)$ with set-up and storage space for long-term experiments as sketched in the floorplan of Fig. 2. Two side laboratories L1 and L2 (machine shop and cleaning facility) and some office space in a $1^{\text {st }}$ floor gallery complement the infrastructure.
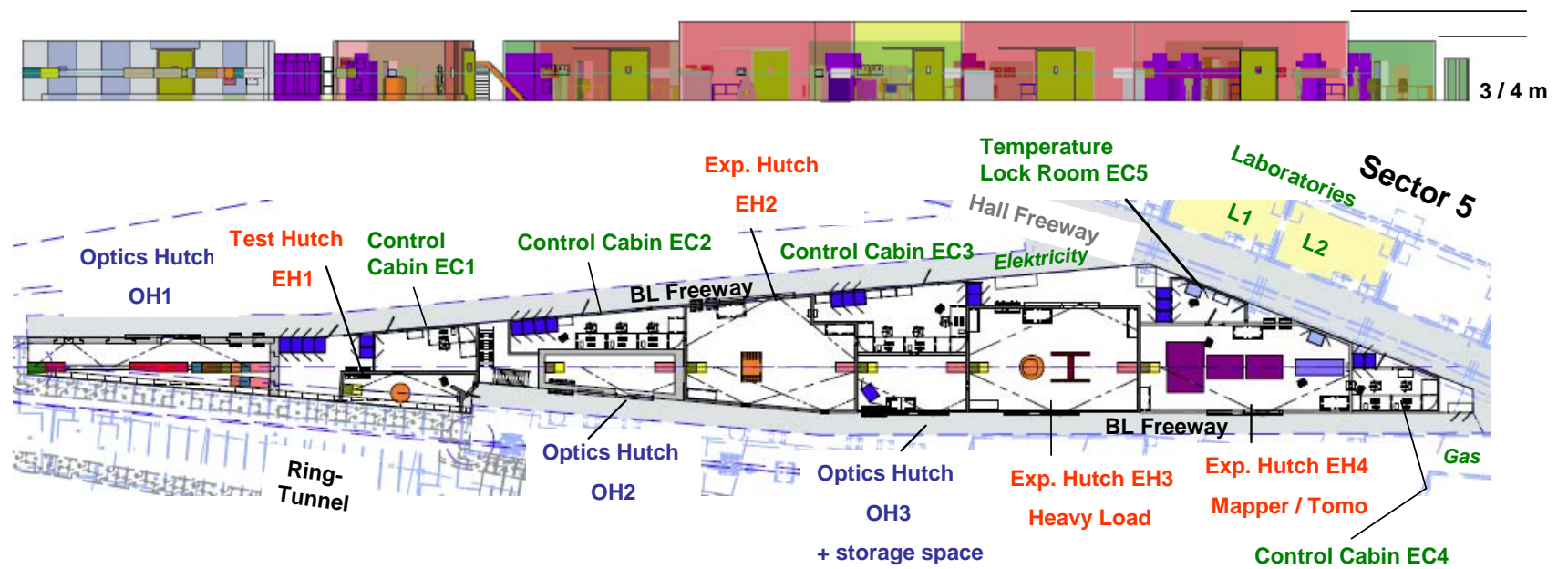

Fig. 2: Side and top view of the current design of HEMS at PETRA III.

Optics. A first optics will consist of water-cooled bent Si(111) Laue crystals ( $40^{\circ}$ asymmetric cut) [5,6] in fixed exit geometry (horizontal deviation $21 \mathrm{~mm}$ ) keeping the beam at $1400 \mathrm{~mm}$ height above the floor. A multilayer filter box inside the ring tunnel cuts out the low-energy harmonics. Lens change-boxes for Compound Refractive Lenses [7] will allow variable focusing with easy handling. In a later stage ML focussing mirrors could be installed in separate optics hutches (OH2, $\mathrm{OH} 3$ ) or substitute the double crystal monochromator in $\mathrm{OH} 1$ ).

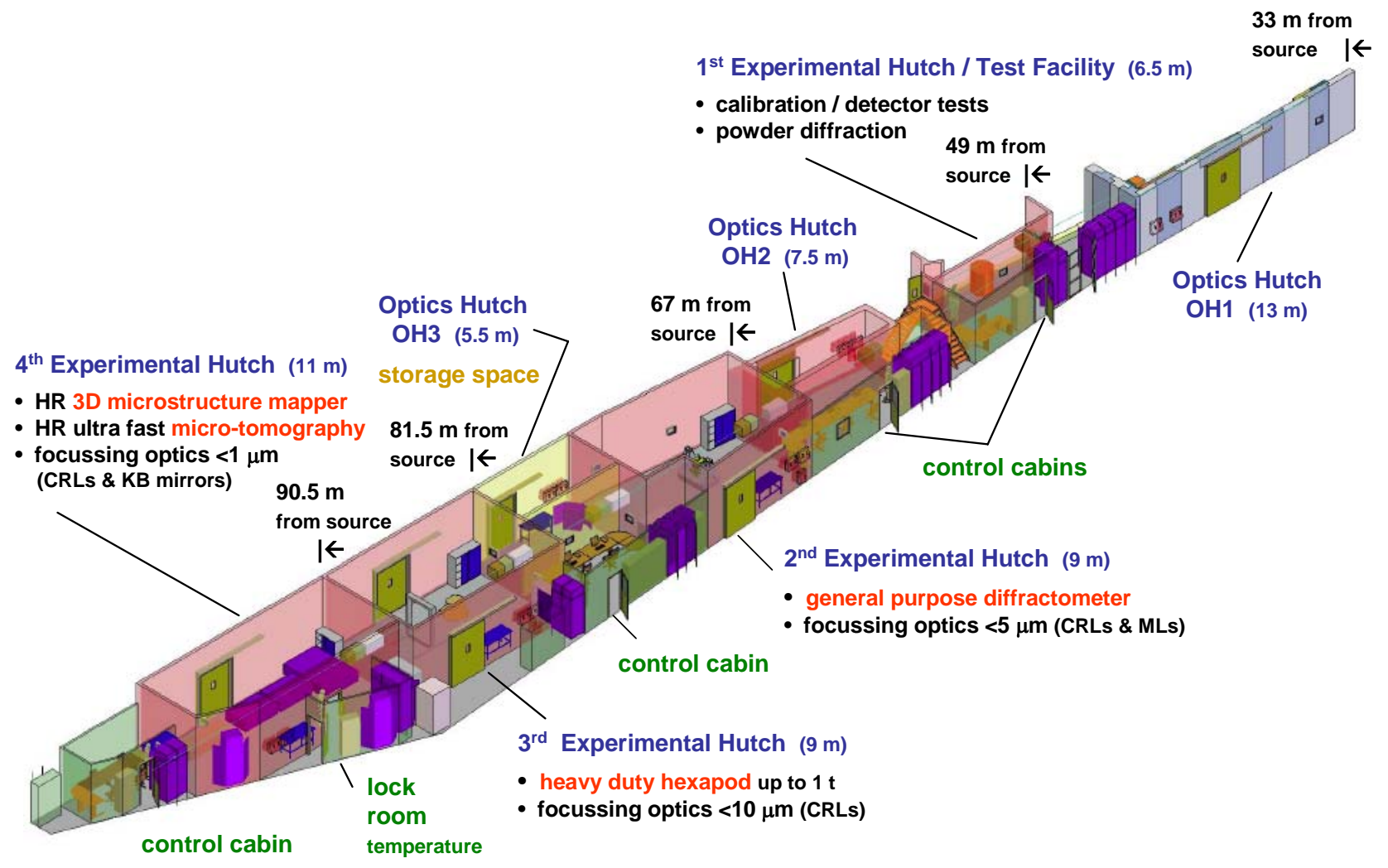

Fig. 3: 3D-view with main experimental stations of HEMS. 
Experiments. The design has been chosen in close collaboration with the future user community and obviously under the constraints of available space, shielding requirements and nature's laws. Still, care has been taken to leave enough space for future upgrades or new scientific fields. Figure 3 gives an overview of the main planned experiments with the possibilities of later modifications.

All experimental stations (radiation shielded hutches) have their neighbouring independent electronic control cabins (EC1, EC2, EC3, EC4), allowing, thus, one group to set up their experiment while another one, further up the beam, is still experimenting with live beam). An inhouse Test Facility permits general testing, detector calibration but also the set-up of minor experiments. This can be done while other experiments are running, however, in a restricted way: a single-bounce monochromator crystal (either Si or Ge, (111), (311) or else) under fixed angle of $4.25^{\circ}$ kicks out a side beam into the small hutch EH1. Any user experiment further down in the direct branch must not be influenced.

Experimental Hutch EH2 allows “general physics” experiments. It will be equipped with a versatile diffractometer from HUBER [8] in an improved design as set-up by H. Reichert et al. [9] at ID15A at ESRF, Grenoble, for the study of deeply buried interfaces. An additional tilt monochromator also enables precise structural investigations of liquid surfaces [10]. The hardware for both instruments including heavy load tables and a scintillaor detector, compare Fig. 4, have been ordered, delivery is expected by spring 2008.

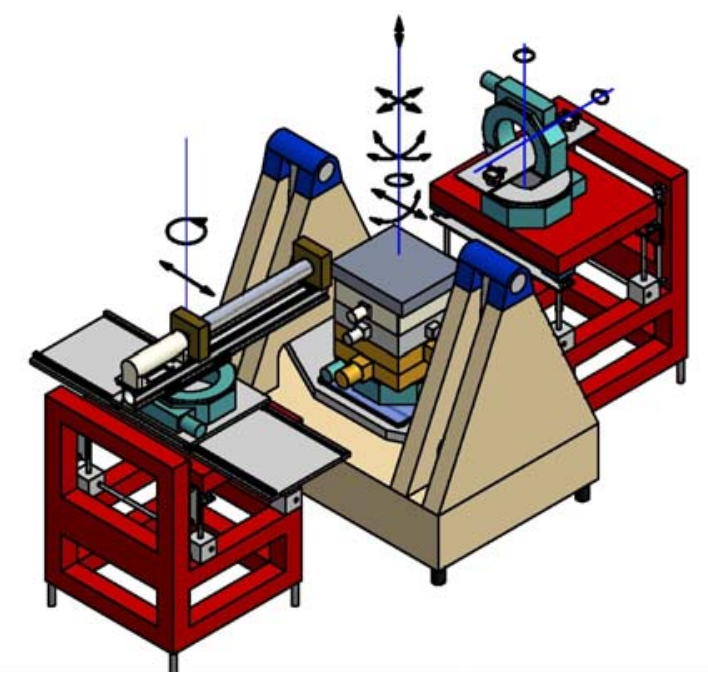

Fig. 4: Modified design for HEMS of a surface diffractometer with pre-tilt-monochromator (courtesy H. Reichert).

Experimental Hutch EH3 will satisfy the engineering community with its demand for handling large and heavy samples (turbine blades and motor blocks) or sample environments (welding and fatigue-loading machines, furnaces and cryostats). Due to the key property of hard $\mathrm{x}$ rays, i.e. a large penetration length, this community can methodically profit from its previous experience in neutron scattering. The much higher brilliance at HEMS, however, will facilitate the construction of corresponding sample-environments, emphasizing in-situ investigations of structural transformations during manufacturing processes. The work-horse will be a prototype hexapod for heavy load of $1 \mathrm{t}$ currently being designed at Physik Instrumente [11] according to demanding specifications (travel ranges tens of $\mathrm{cm}$, tilt angles $15^{\circ}$, resolution $1 \mu \mathrm{m}$ ). Delivery is foreseen also by spring 2008. Figure 5 shows a commercial base-model as a design start together with a typical hydraulic fatigue-loading apparatus from Instron [12]. As 2D-detectors, a mar345 image plate [13] is already on stock, and a mar555 selenium based flat panel direct conversion detector [14] has been tested on the complementary HARWI-II outstation of GKSS at HASYLAB, Hamburg [15]. 

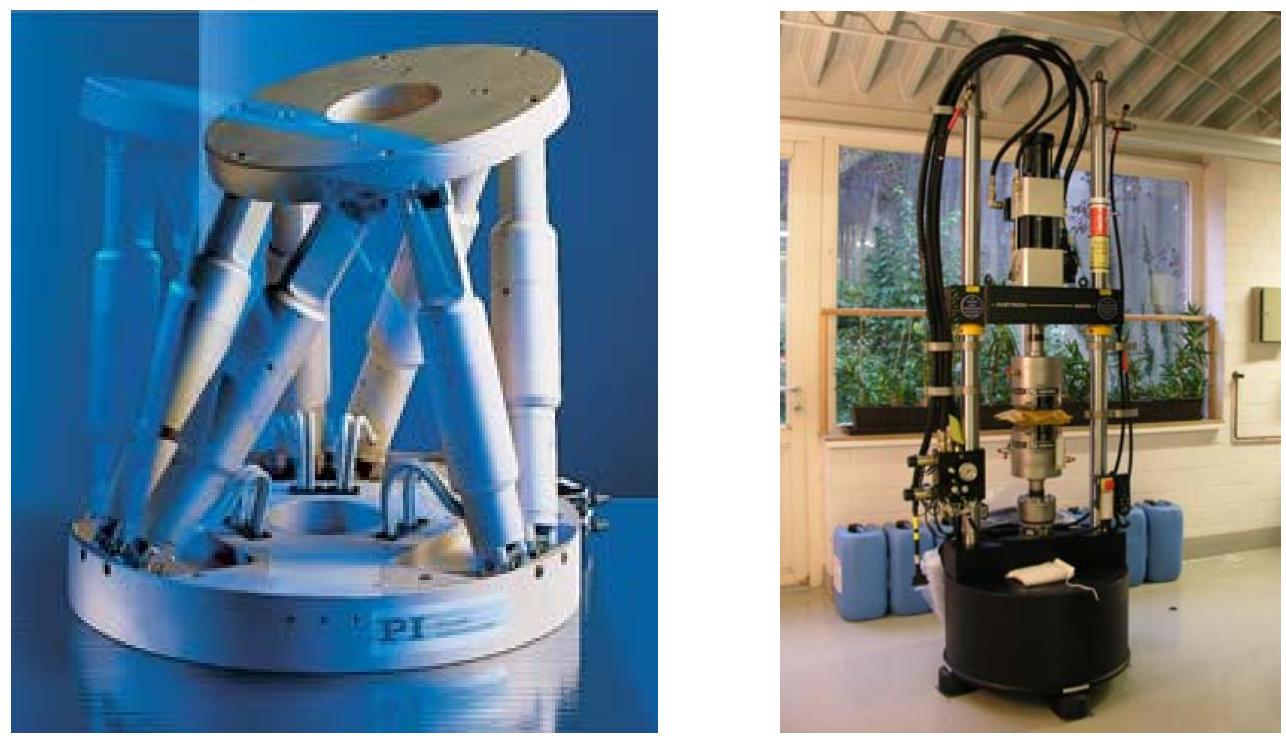

Fig. 5: Base-model M-850 hexapod for $200 \mathrm{~kg}$ load capacity from PI (left), and hydraulic fatigue-loading machine with $+/-100 \mathrm{kN}$ from Instron (right, during commissioning at HARWI), for dual use at HARWI and HEMS.

In the last Experimental Hutch EH4 two specialized instruments will share the separately climatized space (accessible via a climatisation lock room EC5): a 3D-XRD microscope for stress and strain mapping of polycrystalline materials - its principle analogous to the pioneering work of H.F. Poulssen [16], and a dedicated micro-tomography set-up. It will complement the existing setup at the wiggler beamline HARWI-II which is operating in the energy range 16-150 keV and the future Imaging Beamline P05 at PETRA which will operate in the range 5-50 keV. All stations will use rotary air-bearing stages with a wobble of sub-microradians [17]. The location and separate climatisation should allow a stable focus down to the $100 \mathrm{~nm}$ range. Figure 6 shows a first design for the micro-structure mapper.

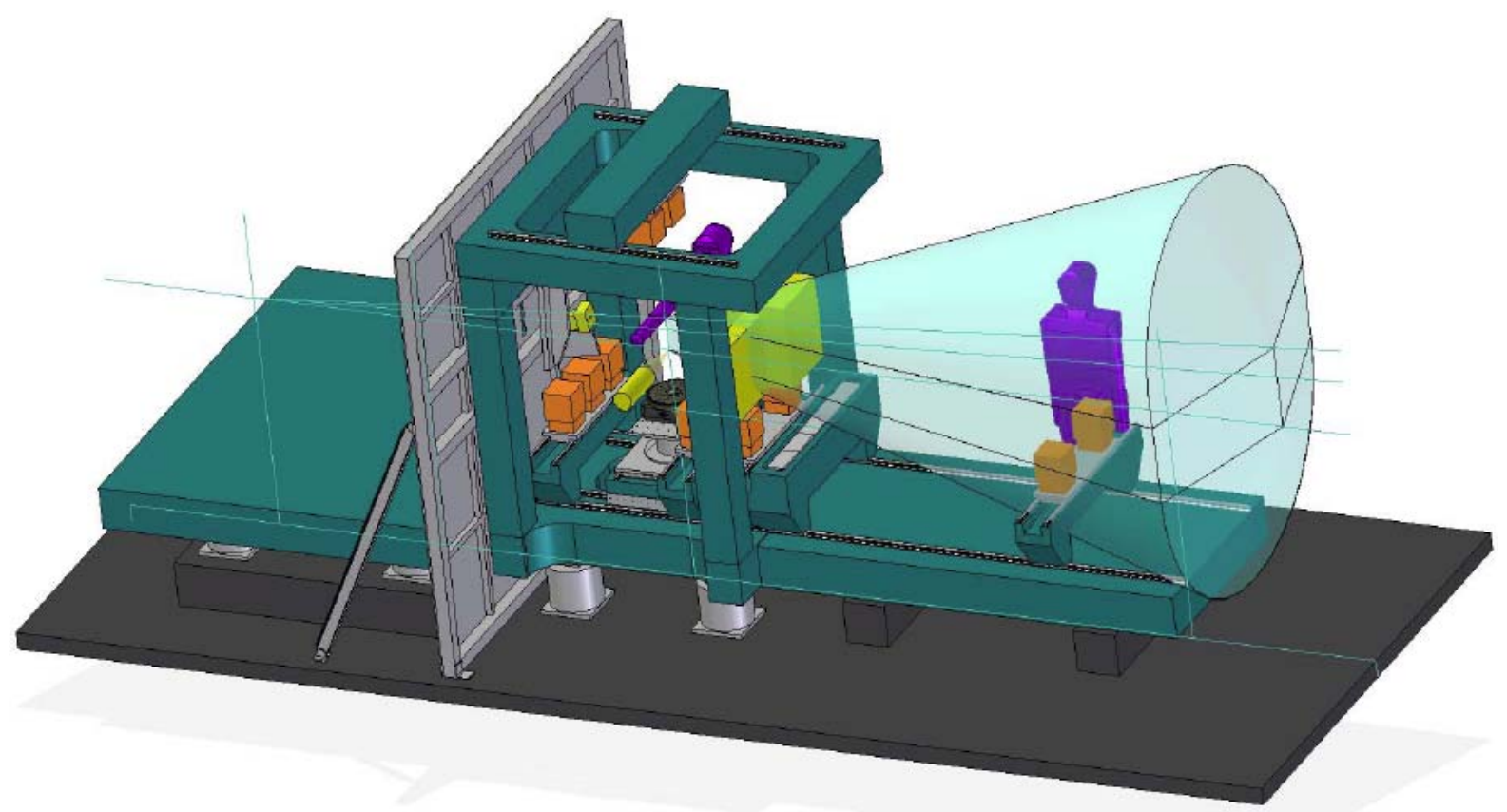

Fig 6: 3D-XRD micro-structure mapper with sample-handling in the center part, shielded optics to the left (with free space shown for Kirkpatrick-Baez Multilayer mirrors or Compound Refractive Lenses) and detector space to the right. 


\section{Summary}

The HEMS beamline at PETRA III is optimized for the key properties of hard x-rays in the fully tunable range 30-300 keV - large penetration depth, negligible extinction and Bragg scattering, large Ewald spheres - to investigate non-destructively bulk properties or deeply buried structures mainly in the context of Materials Research, but versatile enough to allow singular experiments in the merging fields of physics, chemistry and biology. Fast 2D-detectors together with varying focus spots down to $200 \mathrm{~nm}$ in the future will allow highly dynamic in-situ experiments of microstructural transformations. It can handle large and heavy user provided equipment.

Two specialized experiments, currently designed in-house at GKSS, will allow the detailed characterization of grains, their interaction and their stress and strain states with sub-micrometer resolution, as well as tomographic imaging.

Peripheric laboratories will further allow full service also for long-term user experiments on the site. Orders for key components (diffractometers, detectors, shielding hutches) have been placed. An early commissioning of the beamline is foreseen in spring 2009.

\section{Acknowledgement}

We gratefully acknowledge the financial support by BMBF, the encouragement and administrative help of the management at GKSS and DESY, as well as the daily fruitful discussions and exchange of ideas among the PETRA team and our many colleagues at GKSS and HASYLAB.

\section{References}

[1] Information on

http://petra3.desy.de/beamlines/work_packages/e1036/e1557/index_eng.html

[2] R.V. Martins and V. Honkimäki: Textures \& Microstructures Vol. 35 (2003), p. 145

[3] K. Balewski et al. (eds): PETRA III: A Low Emittance Synchrotron Radiation Source (Technical Design Report, Hamburg, DESY, 2004)

[4] Information on http://www.gkss.de/index_e.html

[5] Z. Zhong, C.C. Kao, D.P. Siddons and J.B. Hastings: J. Appl. Cryst. Vol. 34 (2001), p. 504

[6] Z. Zhong, C.C. Kao, D.P. Siddons and J.B. Hastings: J. Appl. Cryst. Vol. 34 (2001), p. 646

[7] Information on http://tudresden.de/die_tu_dresden/fakultaeten/fakultaet_mathematik_und_naturwissenschaften/fach richtung_physik/isp/skm/research/xray_lenses

[8] Information on http://www.xhuber.com/

[9] H. Reichert, V. Honkimäki, A. Snigirev, S. Engemann and H. Dosch: Physica B Vol. 336 (2003), p. 46

[10] V. Honkimäki, H. Reichert, J.S. Okasinski and H. Dosch: J. Synchrotron Rad. Vol. 13 (2006), p. 426

[11] Information on http://www.physikinstrumente.com/

[12] Information on http://www.instron.us/wa/products/default.aspx

[13] Information on http://www.mar-usa.com/products/mar345.htm

[14] Marresearch GmbH, Hans-Böckler-Ring 17, 22851 Norderstedt, Germany

[15] F. Beckmann, T. Dose, T. Lippmann, L. Lottermoser, R.-V. Martins and A. Schreyer: AIP Conf. Proc. Vol. 879 (2007), p. 746

[16] H.F. Poulsen: Three-Dimensional X-Ray Diffraction Microscopy (Berlin, Springer, 2004)

[17] Information on http://www.aerotech.com/products/stages/abrt.html 